\title{
Cooling flows in clusters of galaxies
}

\author{
A.C. Fabian \\ Institute of Astronomy, Madingley Road, Cambridge CB3 0HA, UK
}

\begin{abstract}
The gas temperature in the cores of many clusters of galaxies drops inward by about a factor of three or more within the central $100 \mathrm{kpc}$ radius. The radiative cooling time drops over the same region from 5 or more Gyr down to about $10^{8} \mathrm{yr}$. Although it would seem that cooling has taken place, XMM and Chandra spectra show no evidence for strong mass cooling rates of gas below $1-2 \mathrm{keV}$. Chandra images show holes coincident with radio lobes and cold fronts indicating that the core regions are complex. The observational situation is reviewed here and ways in which continued cooling may be hidden are discussed, together with the implications for any heat source which balances radiative cooling.
\end{abstract}

\section{Introduction}

The gas density within the central $100 \mathrm{kpc}$ or so of the centre of most clusters of galaxies is high enough that the radiative cooling time of the gas is less than $10^{10} \mathrm{yr}$. The cooling time drops further at smaller radii, suggesting that in the absence of any balancing heat source much of the gas in the central regions is cooling out of the hot intracluster medium. In order to maintain the pressure required to support the weight of the overlying gas, a slow, subsonic inflow known as a cooling flow develops.

X-ray observations made before Chandra and XMM-Newton were broadly consistent with the cooling flow picture (see 23 for a review and 42 for an opposing view), although several issues remained unresolved. The first issue was the observed X-ray surface brightness profile, which was not as peaked as expected from a homogeneous flow. Instead a multiphase gas was assumed, dropping cold gas over a range of radii. The second was the fate of the cooled gas. At the rates of $100 \mathrm{~s}$ to more than $1000 \mathrm{M}_{\odot} \mathrm{yr}^{-1}$ found in some clusters, the central galaxies should be very bright and blue if the cooled gas forms stars with a normal intialmass-function. In many cases they do have excess blue light indicative of massive star formation [36, 1, 13, 15], but at rates which are a factor of 10 to 100 times lower than the X-ray deduced mass cooling rate. It has been argued [46] that there is no significant sink in terms of cold gas clouds. A third issue involved the shape of the soft X-ray spectrum, which was inconsistent with a simple cooling flow. Absorption intrinsic to the flow was found to be a possible explanation [2, 3]. A final, major, issue was whether the neglect of heating is justified. The effect of gravitational heating as the gas flows was taken into account, but the effects pf any central radio source, which pumps energy into the surrounding gas via jets, together with disturbances due to subclusters plunging into the core every 
few Gyr were not included due to a lack of quantitative information. Heat flow due to thermal conduction was also generally assumed negligible.

The situation with cluster cooling flows has been clarified over the past year, particularly by the high spatial resolution imaging of Chandra and the high spectral resolution of the XMM-Newton Reflection Grating Spectrometer (RGS). Chandra images show much detail in the cores of clusters, with bubbles from radio sources [41, 24] and cold fronts [40, 57] seen. RGS spectra [50, 54, 38] confirm the presence of a range of temperatures in cooling flow clusters but fail to show evidence for gas cooling below 1-2 keV. Simply put, the data are consistent with gas cooling at a high rate to about one third of the mean temperature beyond $100 \mathrm{kpc}$ but then vanishing.

At about the same time, the evidence for both warm [35,17, 20] and cold [19] molecular gas at the centres of cooling flows clusters has become widespread. In some extreme cases there may be over $10^{11} \mathrm{M}_{\odot}$ of cold gas [19]. The presence of dust in these regions is also widespread, as demonstrated by the Balmer decrement in the optical/UV nebulosities commonly seen (e.g. 32, 15), dustlanes, and submm and IR detections [18, 3, 34]. It is therefore possible that more star formation, and in particular cold gas clouds, may be found in and around central cluster galaxies (see also 29 for a discussion of the properties of very cold gas clouds). There has also been the intriguing detection of OVI emission from A2597 with FUSE [47]. Lastly, recent numerical simulations of evolving cluster which include radiative cooling of the gas predict cooling flows (e.g. 48).

At face value the X-ray data tempt many to assume that some form of heating balances cooling and so dismiss cooling flows altogther. That ignores the how, why and what of the heating, which remains unsolved, although several candidates have been identified $[56,6,16,10,11]$. Some form of feedback is probably required to prevent all of the gas from being heated up. If feedback does occur we have a good chance to observe how it works, since the region is spatially resolved and optically thin. The process is of wide importance, since it provides the upper mass limit for galaxies (in simulations of the galaxy luminosity function, [39] switch off cooling in massive galaxies).

My own view is to treat it as an intriguing astrophysical puzzle whch can be tackled observationally. Heating from radio sources and infalling subclusters must occur at some level, but whether it can balance radiative cooling over the required spatial scales to better than a factor of a few is not yet clear. Cooling probably does account for the observed star formation and cold gas clouds. A major remaining issue is whether the mass cooling rates are reduced from the earlier X-ray deduced rates by a factor of a few, ten or a hundred. We may be witnessing a nearby example of the kind of feedback processes common in galaxy formation; in particular, one in which accretion onto the central black hole and the resultant kinetic energy release play a major role. 


\section{Chandra results}

Chandra images show structure in cluster cores. The X-ray emission is steeply peaked into the centres of many clusters but there are holes and fronts in the peak. Markevitch et al [40] found sharply defined cold fronts on A2142, across which the pressure is continuous yet the temperature changes by a factor of about 2. Ettori \& Fabian [21] note that thermal conduction must be heavily suppressed in order that such sharp features can last long enough to be common. The fronts probably indicate that the gas of subclusters does not readily mix with the existing intracluster medium, presumably because they are separate magnetic structures. Also the cores of infalling subclusters may not be strongly shocked in decelerating into the core (see [44, 22]).

\subsection{The Perseus cluster}

Holes in the X-ray surface brightness are seen to coincide with some radio lobes. The best examples are in the Perseus cluster, and were first seen with ROSAT [7]. Chandra shows that they have bright rims of X-ray cool gas [7]. This is contrary to the work of [33] who predicted that the rims would signify shocks. Other holes coincident with radio lobes are found in Hydra A [41, 16] and many other clusters. The puzzling aspect if radio sources are heating the cooling gas is that in all cases reported the coolest gas seen is that closest to the radio lobes. Of course there is much energy going into the lobes, but the energy from the $P d V$ work expended in forming the holes can propagate away as sound waves, and the relativistic energy stored in the bubbles can be lifted away and out of the immediate core by buoyancy.

Provided that the filling factor of the holes by relativistic plasma is high, then the jet power required to make the holes in the Perseus cluster is considerable at about $10^{45} \mathrm{erg} \mathrm{s}^{-1}[27]$.

\section{$2.2 \quad \mathrm{~A} 1795, \mathrm{~A} 2199$ and the Centaurus cluster}

The Chandra image of A1795 [25] shows an $80 \mathrm{kpc}$ long soft X-ray feature coincident with an $\mathrm{H} \alpha$ filament found by [14]. It is plausibly a cooling wake trailing behind the central galaxy, which is at the head of the filament. The galaxy is moving around in the core of the cluster at a few hundred $\mathrm{km} \mathrm{s}^{-1}$. There is no evidence from the temperatures of the gas that this motion has heated the gas significantly. The central galaxy in A2199 may also be oscillating, as deduced from the unusual morphology of its radio source [12]. Again, the coolest gas appears to be close to both the radio source and the central galaxy [37].

The Centaurus cluster shows a cool, plume-like feature which appears about $20 \mathrm{kpc}$ long [52]. The gas temperature drops from about $3.5 \mathrm{keV}$ down to about $1 \mathrm{keV}$ at the centre, with evidence that more than one phase is present there. Interestingly, the known strong abundance gradient in iron peaks at about 1.5 times Solar at about $15 \mathrm{kpc}$ before dropping back down to about 0.5 near the 

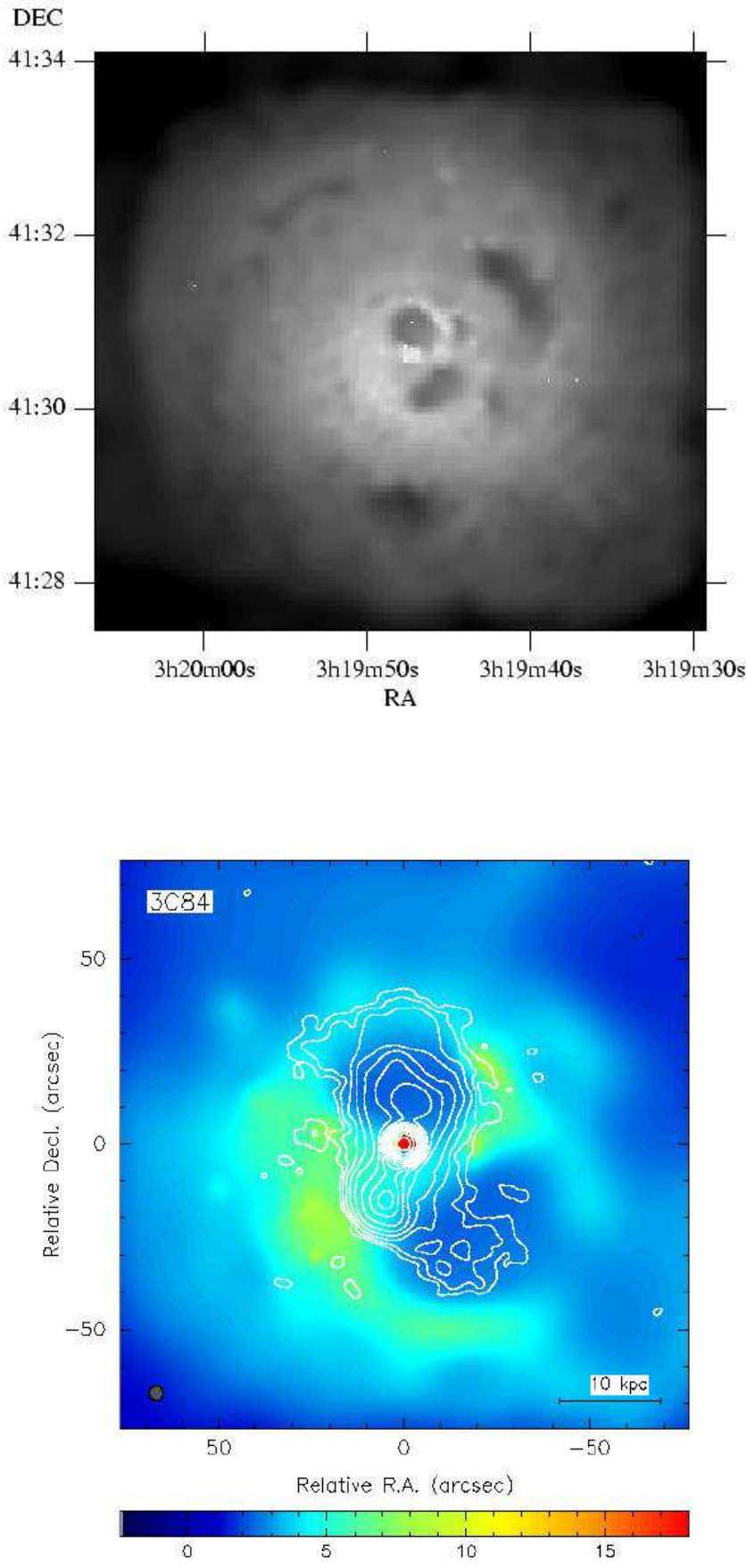

Fig. 1. (Top) Adaptively-smoothed $0.5-7 \mathrm{keV}$ ACIS-S X-ray image of the centre of the Perseus cluster. (Bottom) Radio image (1.4 GHz restored with a 5 arcsec beam, produced by G. Taylor; see [24], overlaid on an adaptively smoothed $0.5-7 \mathrm{keV}$ X-ray map. 

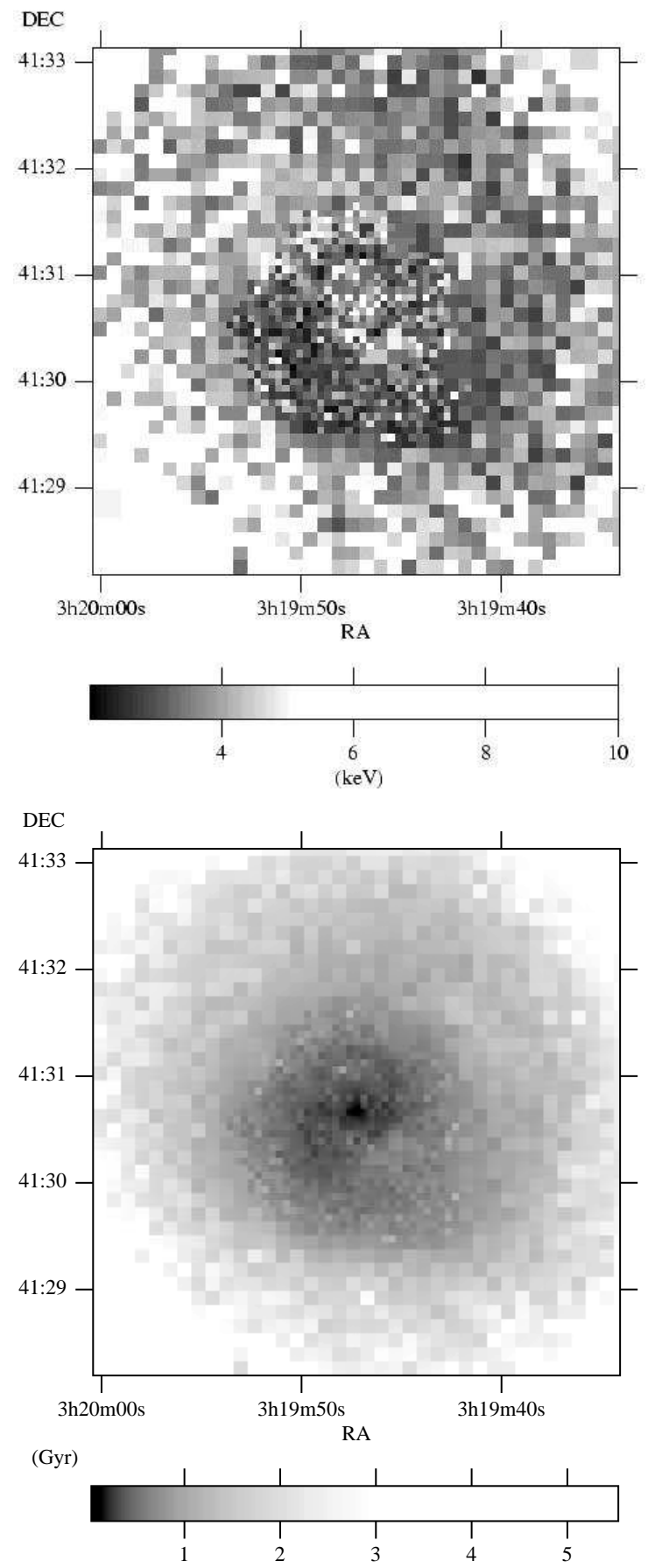

Fig. 2. (Top) Temperature and (Bottom) radiative cooling time maps produced from the X-ray colour ratios [24]. Note that the coolest gas $(T \sim 2.5 \mathrm{keV})$ with the shortest cooling time $(\sim 0.3 \mathrm{Gyr})$ lies in the rim around the $\mathrm{N}$ lobe and in the $\mathrm{E}$ bright blob. Single-phase gas has been assumed for the analysis. 

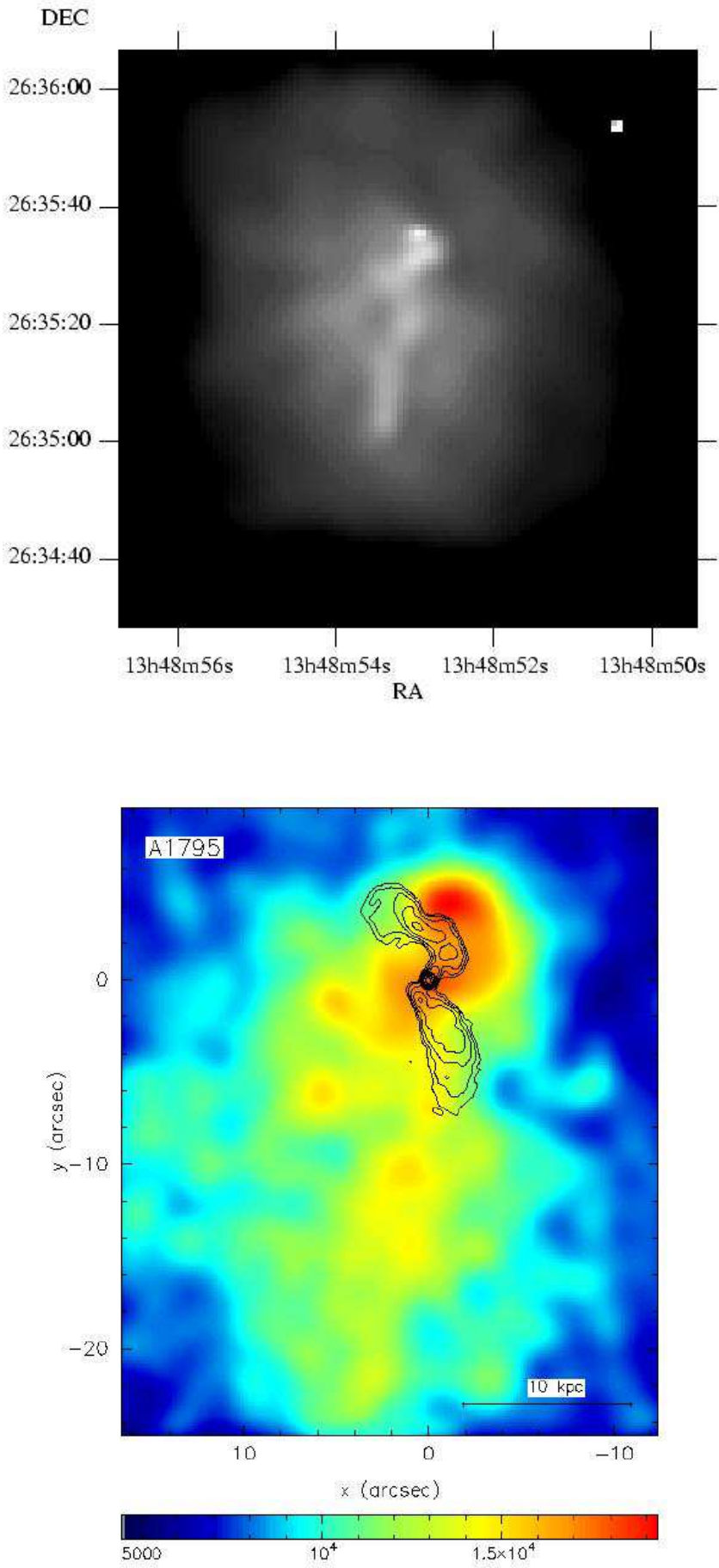

Fig. 3. (Top) Adaptively-smoothed X-ray image of the centre of A1795 [25]. (Bottom) Overlay of the $3.6 \mathrm{~cm}$ radio emission [31] on the X-ray image. 

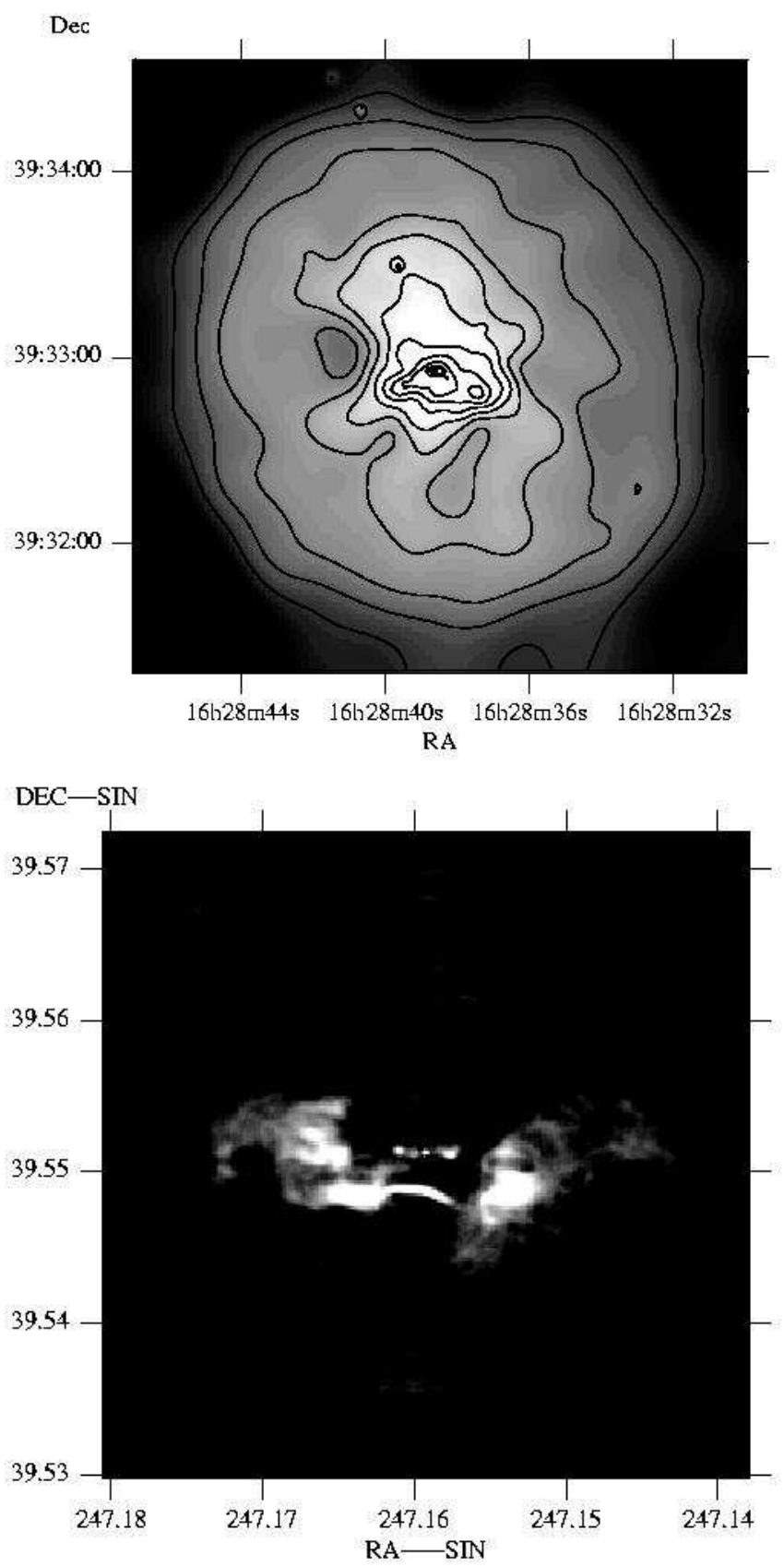

Fig. 4. (Top) Adaptively-smoothed 0.5-3 keV X-ray image of the core of A2199 (the contours are logarithmic); (Bottom) 1.7 GHz radio image [30]. See [37] for analysis of the X-ray image).the X-ray surface brightness drops at the position of the outer radio lobes. 

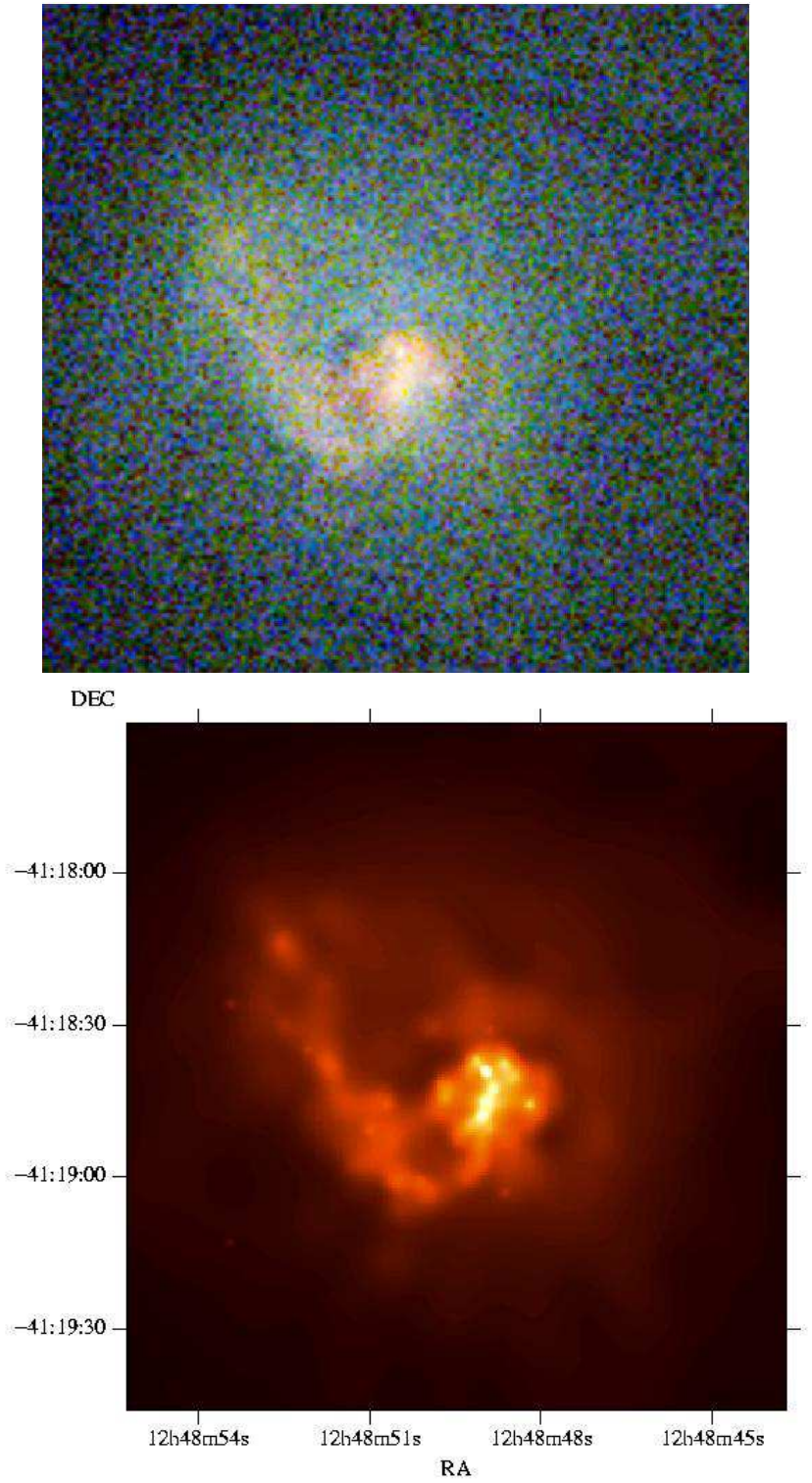

Fig. 5. (Top) Raw colour image and (Bottom) adaptively-smoothed 0.5-3 keV X-ray image of the core of the Centaurus cluster [52]. The radio source in the central cluster galaxy, NGC 4696, has a complex cap-like structure, 30 arcsec wide, fitting around the head of the X-ray plume-like feature [55]. 
centre. This limits the amount of widespread mixing or convection that can have occurred within the core.

In summary, the high-spatial-resolution Chandra data are revealing that cluster cores are complex with a combination of holes due to radio lobes as well as filaments, plumes and cold fronts. All cores studied so far where the radiative cooling time of the gas is a few Gyr show significant central temperature drops.

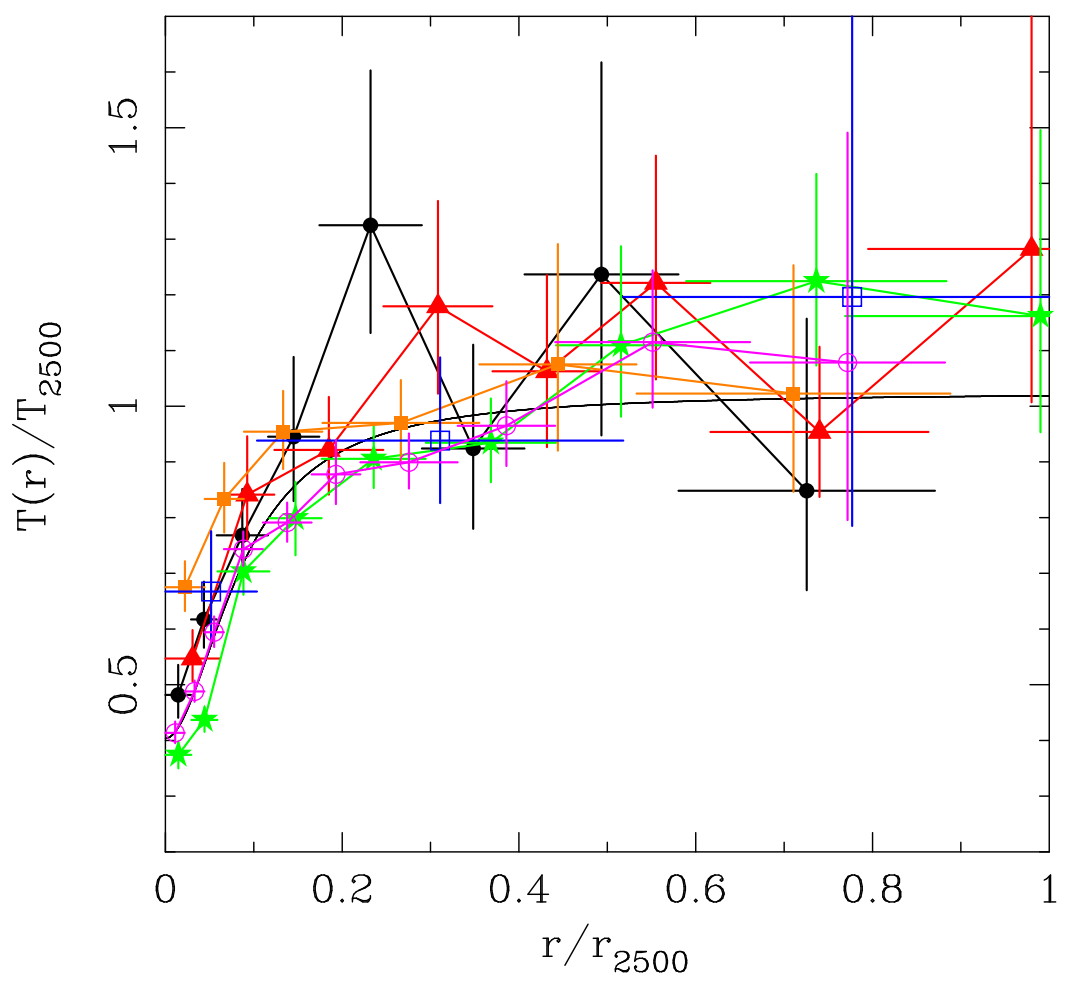

Fig. 6. Chandra temperature profiles for six massive clusters: PKS0745-191, A2390, A1835, MS2137-2353, RXJ1347-1145 and 3C295, from [4]. Note the temperature drop within $\sim 0.2 r_{2500} \sim 120 \mathrm{kpc}$.

\section{XMM-Newton results}

The most striking results have come from the RGS data which show little evidence for gas cooling below 1-2 keV [50, 53, 38]. Emission lines from FeXX and XVII, for example, should be bright and easily seen if the mass cooling rate is high, but they are absent. EPIC CCD spectra (e.g. [9, 43]) confirm this result.

Various explanations have been given [50, 26, 28]. The gas may be cooling and yet appear to vanish when it reaches say $2 \mathrm{keV}$. Clouds of cold gas may 


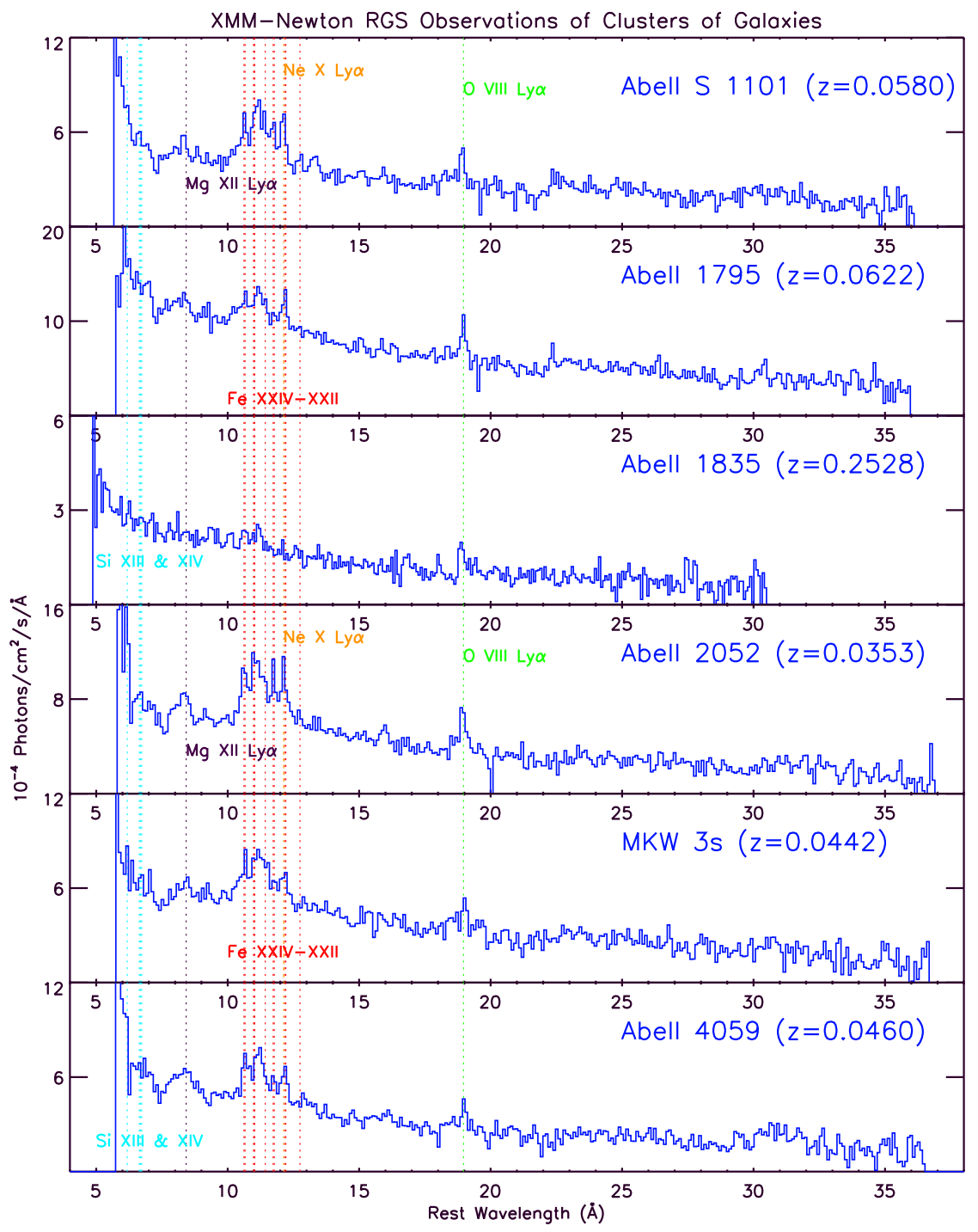

Fig. 7. RGS spectra of 6 cooling flow clusters, kindly provided by J. Peterson and J. Kaastra. Emission lines between 10-13 A indicate the presence of cooler gas in these clusters (at 1-3 keV) but the lack of lines between 13 and 18 A shows that gas is not radiatively cooling below $1-2 \mathrm{keV}$ at high rates in any simple unobscured manner. 
photoelectrically absorb the soft X-rays, or the gas may have become dense enough to separate from the flow and mix in with surrounding hotter gas [45]. Alternatively it may mix in with colder gas (for example that associated with the optical filaments or the molecular gas - note that this also explains why the filaments are so bright; [28]).

Another possibility is that the metals in the gas are not uniformly mixed in, but have a bimodal distribution [26]. Gas in which ten per cent has a metallicity of 3 times solar and 90 per cent has zero metallicity has the same spectrum as gas at 0.3 solar if cooling is unimportant. When it does cool however, the metal emission lines cool only 10 per cent of the gas and so are much reduced as compared with the situation if they were responsible for cooling all the gas.

If heating is the explanation, then it cannot just be some low level of heat which stops the gas at 1-2 keV, since it would then accumulate at that temperature, contrary to observation. It has to halt the cooling over the full range of temperatures, and thus radii. How this can happen is a puzzle. If the radio source is responsible, then it may be intermittent. Maybe we do not see the heating phase, which is short lived. However the power required to stem the flow during the heating phase then goes up to high values. There may not be any problem in the Virgo cluster around M87 where the energy rquirements are relatively small, but in a massive cluster like A1835 [50, 53] the necessary power may exceed $10^{46} \mathrm{erg} \mathrm{s}^{-1}[26]$.

\section{Discussion}

The central $100 \mathrm{kpc}$ radius region in most clusters has a radiative cooling time shorter than 5 Gyr (Peres et al 1997) and many have central cooling times of only $10^{8} \mathrm{yr}$. The gas temperature drops by a factor of 3 or more over this radius range. It seems plausible that the temperature drop and short radiative cooling times are related and that the low temperatures are caused by radiative cooling.

It is then a puzzle as to why gas which has cooled by a factor of three, and for which the radiative cooling time has reduced by a factor of ten or more, is not seen to cool further.

There are two obvious solutions, both of which have some difficulties. The first solution is that the gas does cool but either the soft X-ray emission is absorbed or the cooling is non-radiative and due, say, to mixing. The problem of the fate of cooled gas then remains. The second solution is that some heating balances cooling. The problem here is that the heat has to balance cooling over a wide range of radii and a wide range of timescales. Also observations of radio lobes which are a likely source of heat indicate that they coincide with the coolest gas in cluster cores.

The answer may be more complex, with the major temperature drop being due to a combination of in situ radiative cooling and gas introduced from dense cooling subclusters. Heat from the kinetic energy of infalling subclusters, and turbulence, continues to be dissipated throughout the core, reducing the age of any steady central cooling region to only a few Gyr. An intermittent central radio 
source powered by accretion from the intracluster medium heats and churns up some of the coolest gas at the centre. Radiatively-cooling clumps (possibly metal rich) fall out of the mean slow inflow once their temperature drops to below one third of the outer temperature and rapidly mix with cooler gas clouds closer to the centre. The mixture would have a temperature of about $10^{5} \mathrm{~K}$ (as in mixing layers; Begelman \& Fabian 1990), and rapidly lose its thermal energy by UV emission. Most of this would be absorbed by neighbouring cold gas and dust, to be reradiated as optical/UV line emission and infrared dust emission [28]. Massive star formation take place in cooled clumps and spreads dust into the surrounding gas, further enhancing cooling via infrared emission.

\section{Acknowledgements}

I am grateful to my many collaborators, in particular Steve Allen, Carolin Crawford, Stefano Ettori, Roderick Johnstone, Jeremy Sanders, Robert Schmidt and

Greg Taylor for help and discussions, and the organisers for creating a timely and memorable meeting. The Royal Society is thanked for support.

\section{References}

1. Allen S. W., 1995, MNRAS, 276, 947

2. Allen S.W., Fabian A.C., 1997, MNRAS, 286, 583

3. Allen S. W., Fabian A. C., Johnstone R. M., Arnaud K. A., Nulsen P. E. J., 2001, MNRAS, 322, 589

4. Allen SW, Schmidt RW, Fabian AC 2001, MNRAS, 328, L37

5. Begelman MC, Fabian AC, 1990, MNRAS, 244, 26

6. Binney J., Tabor G., 1995, MNRAS, 276, 663

7. Böhringer H., Voges W., Fabian A.C., Edge A.C., Neumann D.M., 1993, MNRAS, 264, L25

8. Böhringer H., Nulsen P.E.J., Braun R., Fabian A.C., 1995, MNRAS, 274, L67

9. Böhringer H. et al 2000, A\&A, 365, L181

10. Böhringer H., et al 2001, A\&A, in press, astro-ph/0111112

11. Brüggen M Kaiser CR Churazov E Ensslin TA 2001, MNRAS in press, astro$\mathrm{ph} / 0108486$

12. Burns JO, Schwendeman O, White RA, 1983, ApJ, 271, 575

13. Cardiel N., Gorgas J, Arago-Salamanca A., 1998, MNRAS, 298, 977

14. Cowie L.L., Hu E.M., Jenkins E.B., York D.G., 1983 ApJ, 272, 29

15. Crawford C.S., Allen S.W., Ebeling H., Edge A.C., Fabian A.C., 1999, MNRAS, 306, 875

16. David L, Nulsen P.E.J.,McNamara B.R., Forman, W., Jones C., Robertson B., Wise M., 2001, ApJ, 557, 546

17. Donahue M., Mack J., Voit G. M., Sparks W., Elston R., Maloney P. R., ApJ, 2000, 545, 670

18. Edge A. C., Ivison R. J., Smail I., Blain A. W., Kneib J.-P., 1999, MNRAS, 306, 599

19. Edge A. C., 2001, MNRAS, 328, 762

20. Edge A. C., et al 2001, MNRAS, submitted 
21. Ettori S., Fabian A.C., 2000, MNRAS, 317, L57

22. Fabian A. C., Daines S. J., 1991, MNRAS, 252, 17

23. Fabian A.C., 1994, ARAA, 32, 277

24. Fabian A.C., et al 2000a, MNRAS, 318, L65

25. Fabian A.C., et al 2001d, MNRAS, 321, L33

26. Fabian A.C., Mushotzky R.F., Nulsen P.E.J., Peterson J., 2001a MNRAS, 321, L20

27. Fabian A.C., Celotti A Blundell KM Kassim NE Perley RA 2001b, MNRAS submitted, astro-ph/0111418

28. Fabian A.C., et al 2001c, MNRAS submitted

29. Ferland G., Johnstone RM Fabian AC 2001 MNRAS submitted

30. Giovannini G., Cotton W.D., Feretti L., Lara L., Venturi T., 1998, ApJ, 493, 632

31. Ge J.P., Owen F.N., 1993, AJ, 105, 778

32. Heckman T. M., Baum S. A., van Breugel W. J. M., McCarthy P., 1989, ApJ, 338, 48

33. Heinz S., Reynolds C.S., Begelman M.C., 1998, ApJ, 501, 126

34. Irwin JA Stil M Bridges TJ 2001, MNRAS, 328, 359

35. Jaffe W., Bremer M. N., 1997, MNRAS, 284, L1

36. Johnstone R. M., Fabian A. C., Nulsen P. E. J., 1987, MNRAS, 224, 75

37. Johnstone RM Fabian AC Allen SW Sanders JS 2002 MNRAS submitted

38. Kaastra J., et al, 2001, A\&A 365, L99

39. Kauffmann G., Guiderdoni B., White S. D. M., 1994, 267, 981

40. Markevitch M. et al, 2000, ApJ, 541, 542

41. McNamara B. et al, 2000a, ApJ, 534, L135

42. Makishima K. et al, 2001, PASJ, 53, 401

43. Molendi S., Pizzolato F., 2001, ApJ, 560, 194

44. Motl PM Burns JO Loken C Norman ML 2001, BAAS, 198, 9202

45. Norman C., Meiksin A., 1996, ApJ, 468, 97

46. O'Dea C. et al 1994, ApJ, 422, 467

47. Oegerle W. R., Cowie L., Davidsen A., Hu E., Hutchings J., Murphy E., Sembach K., Woodgate B., 2001, ApJ, 560, 187

48. Pearce FP, Thomas PA Couchman HMP, Edge AC, 2000, MNRAS, 317, 1029

49. Peres C. B., Fabian A. C., Edge A. C., Allen S. W., Johnstone R. M., White D. A., 1998, MNRAS, 298, 416

50. Peterson J.A. et al 2001, A\&A, 365, L104

51. Rosner R., Tucker W., 1989, ApJ, 338, 761

52. Sanders J, Fabian AC, 2001, MNRAS submitted, astro-ph/0109336

53. Schmidt R.W., Allen S.W., Fabian A.C., MNRAS, 327, 1057

54. Tamura T. et al 2001, A\&A, 365, L87

55. Taylor GB Fabian AC Allen SW, 2001, MNRAS submitted

56. Tucker W.H., Rosner R., 1983, 267, 547

57. Vikhlinin A., Markevitch M., Murray S.S., 2000, ApJ, 549, L47 